BRIEF

\title{
Proxy Reports About Household Members With Increased Confusion or Memory Loss, 2011 Behavioral Risk Factor Surveillance System
}

\author{
Valerie J. Edwards, PhD; Lynda A. Anderson, PhD; Angela J. Deokar, MPH
}

\begin{abstract}
Suggested citation for this article: Edwards VJ, Anderson LA, Deokar AJ. Proxy Reports About Household Members With Increased Confusion or Memory Loss, 2011 Behavioral Risk Factor Surveillance System. Prev Chronic Dis 2015;12:140427. DOI: http://dx.doi.org/10.5888/pcd12.140427.
\end{abstract}

\section{PEER REVIEWED}

\section{Abstract}

To provide information about the effects of increased confusion or memory loss (ICML) in households in the United States, we describe primary respondents' reports (proxy reports) about another person in their household experiencing ICML, using 2011 Behavioral Risk Factor Surveillance System (BRFSS) data. We used proxy reports on type of assistance needed, effects on functioning in daily activities, and whether confusion or memory was discussed with a health care professional, stratifying by age of the household member with ICML ( $18-50 \mathrm{y}$ vs $\geq 65 \mathrm{y})$. About $3 \%$ ( $\mathrm{n}=$ 3,075 households) of primary respondents reported living with a household member with ICML; 75\% of these household members needed some type of assistance, and nearly $60 \%$ had discussed ICML with a health care professional. Collecting proxy data about individuals in households may help paint a clearer picture of the characteristics of those experiencing cognitive decline and the potential needs of individuals and families.

\section{Objective}

The Behavioral Risk Factor Surveillance System (BRFSS) has collected nationwide data on health risk behaviors and preventive practices associated with the leading causes of morbidity and mortality for more than 20 years (1). In 2011, information on increased confusion or memory loss (ICML) that occurred during the previous 12 months was collected for respondents, and if they themselves did not report ICML, they served as a proxy for a household member who was reported to be experiencing ICML. Because of initial concerns about self-disclosure of ICML, data were collected about other household members to provide a more comprehensive picture of ICML. The objective of this study was to describe the characteristics of US adults with ICML reported by proxy.

\section{Methods}

Data were included from 13 states (Arkansas, Florida, Georgia, Hawaii, Illinois, Iowa, Louisiana, New Hampshire, North Carolina, South Carolina, Tennessee, West Virginia, and Wisconsin) that had available household weights and included ICML questions on their 2011 surveys (2). Primary respondents who were randomly selected via BRFSS protocol were asked the following screening question: "Have you experienced confusion or memory loss that is happening more often or is getting worse" (yes or no)? If more than 1 adult lived in the household, the primary respondent was also asked "How many adults in your household experienced confusion or memory loss that is happening more often or is getting worse during the past 12 months?" If the primary respondent answered no to the ICML screener $(n=81,012)$ but reported that someone else in the household experienced ICML, the primary respondent began serving as the proxy respondent and answered the 6 ICML questions about the household member with ICML $(\mathrm{n}=3,075)$. When multiple household members experienced ICML, the proxy answered questions about the person with the most recent birthday. Age data for 30 household members with ICML were not available, and these records were excluded from further analyses; the final analytic sample $(\mathrm{n}=3,045)$ was stratified by age ( $18-50$ y vs $\geq 65$ y) (Figure). 


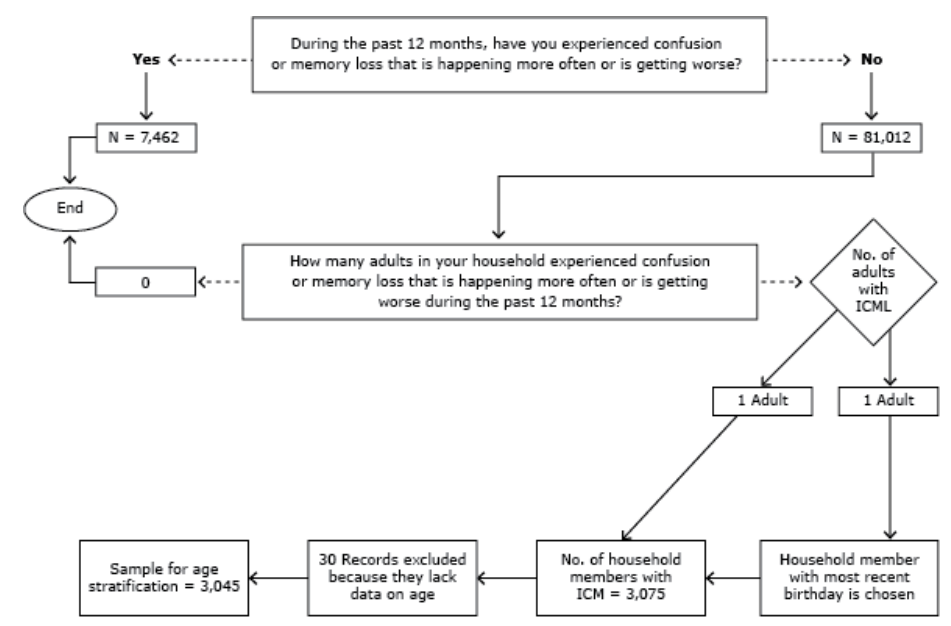

Figure. Classification of BRFSS respondents experiencing ICML themselves or serving as a proxy for another household member experiencing ICML, 2011. The questions on ICML were introduced with the following statement: "The next few questions ask about difficulties in thinking or remembering that can make a big difference in everyday activities. This does not refer to occasionally forgetting your keys or the name of someone you recently met, which is normal." Abbreviations: BRFSS, Behavioral Risk Factor Surveillance System; ICML, increased confusion or memory loss.

Data were weighted using household weights based on the number of occupied households per state, derived from the American Community Survey Public Use Microdata Sample (PUMS) from the 2010 US Census (3). Household weights were used because household members are "nested" within households and the data do not conform to the random selection process used to compute individual-level weights.

Functional difficulties were assessed by asking the proxy whether ICML caused the household member to give up household activities or chores they used to do or interfered with their ability to work, volunteer, or engage in social activities ("always," "usually," or "sometimes" vs "rarely" or "never"). Additional questions asked about type of assistance most needed, if any, and if confusion or memory loss was discussed with a health care professional. Analyses were performed with SPSS version 22 (IBM Corp) to obtain weighted point estimates. Pairwise deletion was used for all analyses.

\section{Results}

There were 3,075 proxies (weighted estimate, 3.7\%; 95\% confidence interval [CI], 3.5\%-3.9\%) who reported about a household member who experienced ICML. For the 3,045 household members with ICML for whom age information was available, about $74 \%$ were aged 60 years or older $(60-69$ y, 19.4\%, 95\% CI,
$18.2 \%-20.6 \%$; 70-79 y, 23.3\%, 95\% CI, 22.1\%-24.6\%; 80-89 y, $23.3 \%, 95 \% \mathrm{CI}, 22.1 \%-24.6 \%$; and aged $\geq 90 \mathrm{y}, 8.1 \%, 95 \% \mathrm{CI}$, $7.4 \%-8.9 \%)$. About one-quarter were aged 18 to $59(18-29 \mathrm{y}$, $2.0 \%, 95 \%$ CI, 1.8\%-2.2\%; 30-39 y, 3.8\%, 95\% CI, 3.4\%-4.2\%, $40-49 \mathrm{y}, 7.5 \%, 95 \% \mathrm{CI}, 6.8 \%-8.3 \%$, and $50-59 \mathrm{y}, 12.6 \%, 95 \% \mathrm{CI}$, $11.7 \%-13.5 \%)$.

For household members aged 18 to $59,68.5 \%(n=463)$ were reported to need some type of assistance in 1 of 5 areas compared with about $76.5 \%(n=1,711)$ of those aged 60 or older (Table). Household members aged 18 to 59 were reported to need the most assistance for household tasks; transportation was reported to require the most assistance for those aged 60 or older. In the area of functional difficulties, $51.9 \%$ of proxies reported that the household member had to give up household chores as a result of ICML, and $52.4 \%$ had given up work, volunteering, or social activities because of ICML, with the older adults having significantly more difficulties than younger adults. Finally, 59.0\% of household members were reported by proxies to have discussed confusion or memory loss with a health care professional; significantly more of the older age group were reported to have had such discussions compared with the younger age group.

\section{Discussion}

The BRFSS collected information from a primary respondent about another adult in a household for the first time in 2011. BRFSS data on children have been collected by randomly selecting a child in the household and obtaining information about that child from the primary respondent. This approach is a reliable way to obtain information on the health status of children who would not normally be included in BRFSS (4). In our study, data collected from proxies allowed us to obtain information about another household member experiencing ICML when the primary respondent did not report experiencing it. Our findings complement other recent findings on functional difficulties among people selfreporting ICML (5) and findings on ICML for entire households (2).

Household members whose ICML is reported by proxy respondents appear to be more impaired by ICML than are respondents who report their own ICML. For example, we found that nearly three-quarters of household members aged 60 or older were reported by proxies to need some type of assistance. In contrast, a recent study using BRFSS data reported that for primary respondents aged 60 or older, only about half reported needing assistance because of ICML (6). However, we note that the proxy data and the self-reported data are not directly comparable because of dif-

\footnotetext{
The opinions expressed by authors contributing to this journal do not necessarily reflect the opinions of the U.S. Department of Health and Human Services, the Public Health Service, the Centers for Disease Control and Prevention, or the authors' affiliated institutions.
} 
ferent weights. These findings also support the importance of examining information about the impact of diminished functioning among others in the household with reported ICML.

Limitations to these findings should be considered. Data on ICML are self-reported and are distinct from data on the prevalence of Alzheimer's disease and related dementias. BRFSS respondents were drawn from households with a telephone landline, thereby limiting the population to those who can afford one and to those who do not live in institutionalized settings. Additionally, data cannot be construed as a national average. Furthermore, research indicates selective bias by age in over- and underreporting disabilities for others (7). Collecting data from proxies about household members may help paint a clearer picture about the characteristics of those experiencing cognitive decline and the potential burden faced by individuals and their families.

\section{Acknowledgments}

The findings and conclusions in this report are those of the authors and do not necessarily represent the official position of the Centers for Disease Control and Prevention

\section{Author Information}

Corresponding Author: Valerie J. Edwards, PhD, Centers for Disease Control and Prevention, 4770 Buford Hwy NE, Mailstop K-45, Atlanta, GA 30341. Telephone: 770-488-8008. Email: vae2@cdc.gov.

Author Affiliations: Lynda A. Anderson, Centers for Disease Control and Prevention, Atlanta, Georgia, and Emory University, School of Public Health, Atlanta, Georgia; Angela J. Deokar, Centers for Disease Control and Prevention, Atlanta, Georgia.

\section{References}

1. Mokdad AH. The Behavioral Risk Factors Surveillance System: past, present, and future. Annu Rev Public Health 2009;30(1):43-54.

2. Deokar AJ, Bouldin ED, Edwards VJ, Anderson LA. Increased confusion and memory loss in households, 2011 Behavioral Risk Factor Surveillance System. Prev Chronic Dis 2015; 12:E29.

3. US Census Bureau. American Community Survey. http:// www.census.gov/acs/www/data_documentation/ public_use_microdata_sample/. Accessed March 16, 2015.
4. Pierannunzi C, Hu SS, Balluz L. A systematic review of publications assessing reliability and validity of the Behavioral Risk Factor Surveillance System (BRFSS), 2004-2011. BMC Med Res Methodol 2013;13(1):49.

5. Anderson LA, Deokar A, Edwards VJ, Bouldin ED, Greenlund KJ. Demographic and health status differences among people aged 45 or older with and without functional difficulties related to increased confusion or memory loss, 2011 Behavioral Risk Factor Surveillance System. Prev Chronic Dis 2015;12:E30.

6. Centers for Disease Control and Prevention. Self-reported increased confusion or memory loss and associated functional difficulties among adults aged $\geq 60$ years -21 states, 2011 . MMWR Morb Mortal Wkly Rep 2013;62(18):347-50.

7. Todorov A, Kirchner C. Bias in proxies' reports of disability: data from the National Health Interview Survey on disability. Am J Public Health 2000;90(8):1248-53.

\footnotetext{
The opinions expressed by authors contributing to this journal do not necessarily reflect the opinions of the U.S. Department of Health and Human Services, the Public Health Service, the Centers for Disease Control and Prevention, or the authors' affiliated institutions.
} 


\section{Table}

Table. Proxy Reports of Problems and Health Care Activity for Household Members With Reported ICML, 2011 Behavioral Risk Factor Surveillance System ${ }^{\mathrm{a}}$

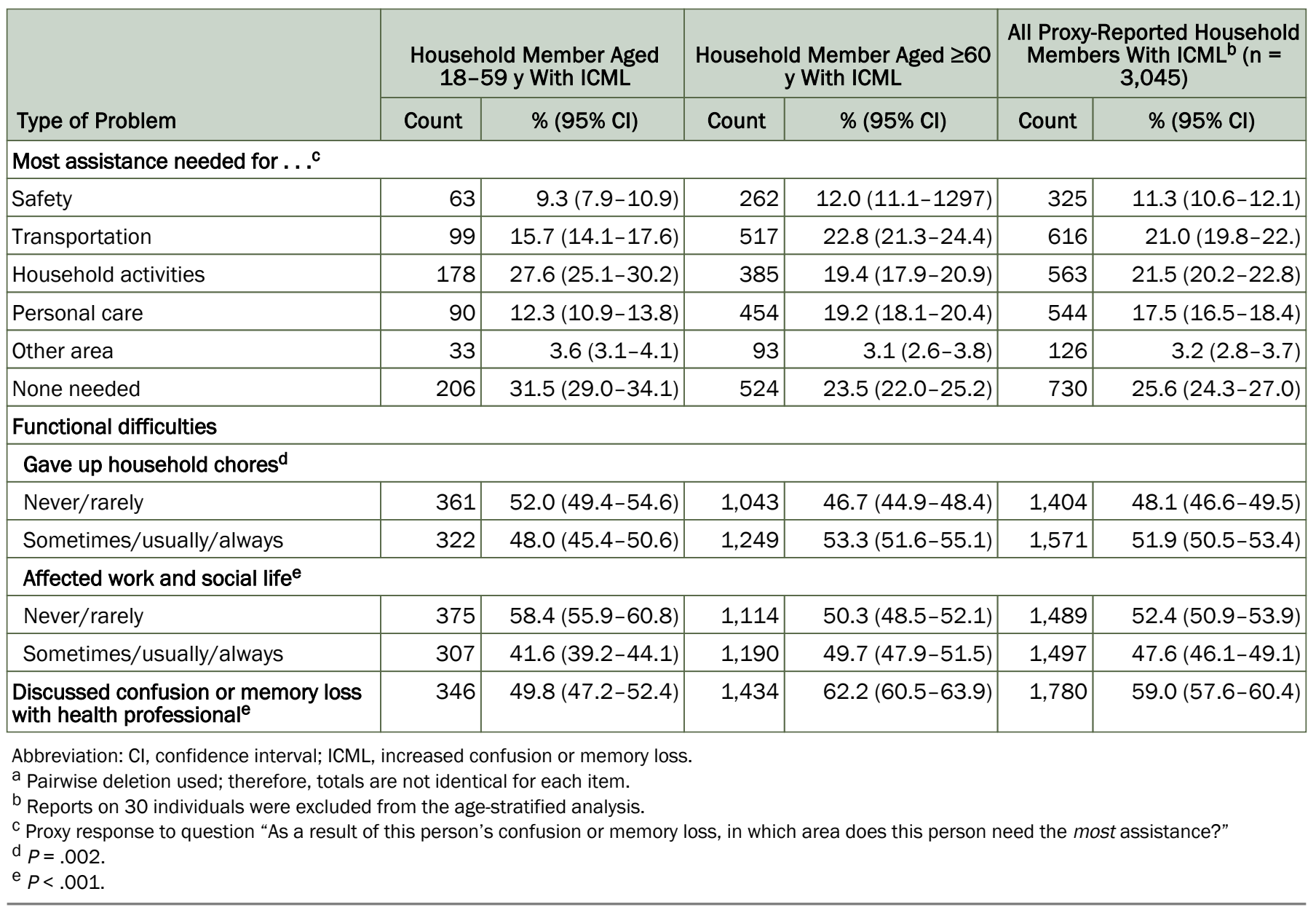

The opinions expressed by authors contributing to this journal do not necessarily reflect the opinions of the U.S. Department of Health and Human Services, the Public Health Service, the Centers for Disease Control and Prevention, or the authors' affiliated institutions. 